Review Article

\title{
The current status of inguinal lymph node dissection for penile cancer in P R of China
}

Jiang-Ping $\mathrm{Du}^{1}$, Nirmal Lamichhane ${ }^{2}$

Department of Urology and Andrology, Affiliated Hospital of Hebei University of Engineering, PR China ${ }^{1}$

Department of Surgical Oncology, Urology Unit, B. P. Koirala Memorial Cancer Hospital, Bhagalpur, Nepal ${ }^{2}$

\section{ABSTRACT}

With the advancement of technology, the inguinal lymph node dissection for penile cancer has developed rapidly. In this paper, the literature published and indexed on CNKI was searched in the past 10 years. The current status of inguinal lymph node dissection for penile cancer was described in terms of surgical trends, timing of surgery, surgical methods, and surgical decision-making.

Keywords: Penile cancer; Inguinal lymphadenectomy; Status in China

\section{Introduction}

With advances in diagnostic technology, improved surgical methods, the use of laparoscopy, new radiotherapy methods, chemotherapeutic drugs, and the use of targeted drugs, the treatment of penile cancer has also achieved unprecedented advancement. The overall surgical concept of inguinal lymphadenectomy for penile cancer has been innovated, from selective surgery to recommended surgery, from open surgery to laparoscopic surgery, from multi-channel to singlechannel laparoscopic surgery, from laparoscopic surgery to robotic surgery, and from staged surgery to simultaneous surgery. In this paper, the literature published on CNKI was searched. The current status of inguinal lymph node dissection for penile cancer was described in terms of surgical trends, timing of surgery, surgical methods, and surgical decision-making.

\section{The trend of surgery}

Currently in China, video endoscopic inguinal lymphadenectomy (VEIL) is the preferred surgical procedure for the treatment of penile cancer. Gradually from the provincial hospitals to municipal hospitals, and even some county-level hospitals, it is also carrying out this technique. Based on CNKI, with the keywords of 'penile cancer inguinal lymph node dissection', 90 related articles were retrieved from January 1, 2008 to December 31, 2017. The measurement and visualization analysis showed that the leading institutions, West China Hospital of Sichuan University and People's Hospital of Zhejiang
Province, had 5 articles respectively (Figure 1). The peak in the literature was reported in 2013, which is 16 (Figure 2). VEIL or/and laparoscopic pelvic lymphadenectomy is a trend in the treatment of penile cancer.

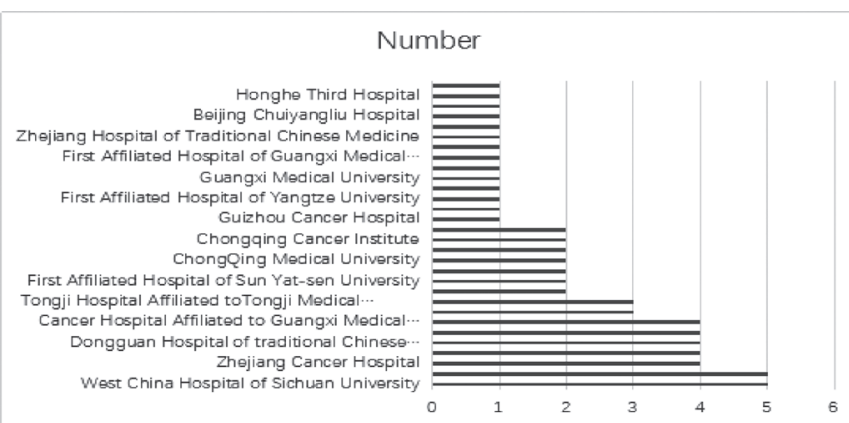

Fig. 1 Distribution of Literature in terms of Institutions in inguinal lymph node dissection for penile cancer during the past ten years.

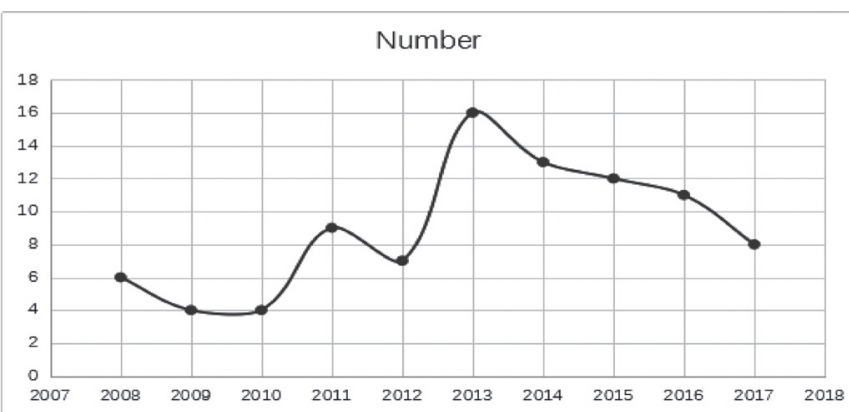

Fig. 2 Trend of Annual literature in inguinal lymph node dissection for penile cancer in ten years.

Correspondence

Dr. Jiang-Ping Du, Department of Urology and Andrology

Affiliated Hospital of Hebei University of Engineering, PR China. E-mail:dujiangping2004@126.com 


\section{Timing of surgery}

Penile lymphography showed a fixed drainage pattern from the superficial to the deep part of the groin area and then to the pelvic lymph nodes. There was no evidence of a "Skip" drainage. Lymphatic drainage at all levels communicates with each other, so the lymphatic drainage of the penis leads to the bilateral groin area. When Iliac, para-aortic, pre-caval and paracaval lymph nodes are invaded, then the disease is considered to be a systemic metastasis. The biological manifestation of squamous cell carcinoma is a long period of time from one region to another before distant metastasis, which provides a favorable therapeutic opportunity for lymphadenectomy.

Local lymph node metastases can be cured. Regional lymph node dissection determines long-term survival. Modified lymph node dissection is recommended for patients with no palpable lymphadenopathy, and standard lymph node dissection can be expanded once positive lymph nodes are found. Patients with positive lymph nodes must undergo bilateral inguinal lymph node dissection. Patients with two or more positive inguinal lymph nodes should also undergo pelvic lymph node dissection.

At present, major guidelines at home and abroad have pointed out that the metastasis of inguinal lymph nodes is an independent risk factor that affects the prognosis of penile cancer. Therefore, inguinal lymph node dissection is highly valued by urologists. In order to reduce the incidence of complications after prophylactic lymphadenectomy and improve the prognosis of patients, urologists try to perform various exploratory studies ${ }^{1}$.

\section{The surgical method}

\section{Inguinal lymph node dissection for penile cancer} Open inguinal lymphadenectomy (OIL) has gradually decreased, and hospitals around the world are more likely to develop VEIL. VEIL is a safe and effective minimally invasive technique that can reduce postoperative complications, especially flap necrosis, incision dehiscence and incision infection ${ }^{2}$.

\section{Video endoscopic inguinal lymphadenectomy for penile cancer $^{3}$}

2.1 Preoperative preparation: Routinely assess the patient's general condition and control the underlying disease; treat penile lesions with $0.1 \%$ benzalkonium bromide or potassium permanganate solution (1:5000) for 2 to 3 days, 3 to 4 times a day; fasting for 8 hours before surgery. Prepare perineal skin. Anesthesia and posture: After general anesthesia, supine position was taken, both lower extremities were abducted by 30 degrees, and the knee joint was bent by 90 degrees. The surgeon and assistant stand on both sides of the lower extremities, and the monitor was placed on the patient's head. Site was prepared and drapped, sterile glove was used to wrap the penile lesions.

2.2 The establishment ports and pneumocutaneum: In the body surface marking of the intersection of sartorius muscle and long adductor muscle, about $3 \mathrm{~cm}$ below, about $10 \mathrm{~mm}$ incision is made and deepened up-to subcutaneous fat and fascia. Blunt dissection is done with finger below the superficial fascia to create a space to enter a visual retroperitoneal dilator and it was gradually inflated with approximately $600 \sim 800 \mathrm{ml}$ of air. During inflation, the balloon dilator is inspected with scope to further verify the correct plane. After inflating for $3 \sim 5 \mathrm{~min}$, the first $10 \mathrm{~mm}$ Trocar is placed at cross section of medial margin of the sartorius and the medial margin of the adductor muscle. Another two 5 and 12 $\mathrm{mm}$ trocars are placed $8 \sim 10 \mathrm{~cm}$ above in both sided from the first Trocar. The first trocar was connected to insufflator machine. The pneumo pressure was set at about $12 \sim 15 \mathrm{mmHg}$, and the established subcutaneous space was confirmed again by putting the 30 degrees scope via $10 \mathrm{~mm}$ Trocar.

2.3 Dissection range: The entire dissection range is 2 $\mathrm{cm}$ above the inguinal ligament, the medial border of the sartorius is the lateral boundary, the lateral border of the long adductor muscle is the inner boundary, and the tip of the femoral triangle is the lower border. At the same time, the scope of dissection involves braces, and the lymphatic adipose tissue of both superficial level and deep level around the great saphenous vein.

2.4 The specific operation process: Enter the camera telescope to confirm the correct level, and identify the fascia lata, the medial edge of the sartorius muscle and the lateral edge of the adductor muscle and other anatomical landmarks. Free the fat and fascial tissue, and then look for saphenous vein. Along the medial edge of the sartorius muscle on the outside, the anterior superior spine is dissociated. Remove upper and lower lateral lymph nodes around the circumflex superficial vein and 
lateral femoral vein. The medial side of the long adductor muscle is detached toward the spermatic cord, and the upper medial lymph nodes of the superficial venous and superficial abdominal veins are removed. In the middle, along the great saphenous vein, the saphenous vein is divided and the lower medial lymph node around the great saphenous vein is removed. At the saphenous vein hiatus, the major branch of the saphenous vein is divided, the central lymph node at the junction of the great saphenous vein and the femoral vein is removed. Absorbable clips were used for ligating the saphenous vein at a distance of $0.5 \mathrm{~cm}$ from it merges into the femoral vein. At the apex of the femoral triangle, the fascia lata was opened, and the femoral vessels were freed and dissected along the femoral vessels. The deep inguinal lymph nodes in the medial and lateral femoral veins, and the lymph nodes in the femoral artery are mainly removed, and the medial lymph nodes in the femoral vein are mainly removed. Cleared lymph node tissue is removed from the $12 \mathrm{~mm}$ cannula. Full hemostasis to the wound is done. The surgical area is sprayed with methylene blue, and it is judged whether there is a spill of milky lymph in the blue background, and the unclosed lymphatic vessels are again consealed and clipped. Indwelling drainage tube was kept at the lowest trocar and connected to the negative suction. Wounds were sutured, elastic compression bandage applied to eliminate the subcutaneous dead space. Another groin is dealt in the same way as of this method described. After the lymph nodes were dissected and the wound protected, partial penectomy or total penectomy was performed.

2.5 Postoperative management: After 2 to 3 days, patient was encouraged to get out of bed. After 2 to 3 days, dressing was changed, Cutaneous blood flow and subcutaneous seroma collection if inspected was managed. If the drainage tube is less than $30-40 \mathrm{ml}$, drainage tube was removed. In particular, if wounds have necrotic skin flaps, alginate dressings can be tried on wet methods. ${ }^{4}$

\section{Preservation of great saphenous vein}

Laparoscopic treatment of penile cancer is similar to open groin surgery, but VEIL can effectively reduce the incidence of postoperative complications. And compared with conventional VEIL and OIL, VEIL with preserving great saphenous vein in penile cancer does not prolong the operation time and also do not increase the difficulty of surgery), does not increase the length of stay and does not affect the yield of lymph node numbers. At the same time, it can significantly reduce postoperative wound infection, seroma formation, lower limb edema and other complications, and is a safe and effective surgery. ${ }^{5}$ The preservation of the large saphenous vein trunk during the operation can effectively prevent the occurrence of postoperative edema of the lower extremities. For those with large saphenous vein branches, other branches may be appropriately ligated. Preservation of the great saphenous vein is the overall development trend during VEIL for penis cancer.

\section{Resection of fascia lata}

The classic OIL surgery requires removal of fascia lata, but in modified OIL, it is conserved. OIL, whether classic or modified, has a relatively high incidence of complications, especially flap ischemia, infection, and necrosis. When those happen, the femoral vessels that have lost the fascial coverage will be directly exposed to the operative field. Therefore, after the removal of the fascia lata, the classical surgical technique was performed by transposing the sartorius muscle on the surface of the femoral vessels to replace the protective effect of the fascia lata. The modified surgical procedure is recommended to retain the fascia lata in order to reduce the occurrence of complications. However, with the development of VEIL, flap-related complications have been theoretically eliminated, and the risk of exposure of the femoral vessels has also been resolved. Thoroughly removing the fascia lata covered on the surface of the femoral triangle can open the femoral sheath and complete the exposure of the posterior border and lymphatic dissection. At the same time, the boundary between the sartorius muscle and the adductor muscle can be clearly revealed, and it can contribute to the location of the outer boundary ${ }^{6}$. The removal of fascia lata is one of the tumor control advantages of VEIL.

\section{Intra-operative lymph node tracing}

Sentinel lymph node (SLN) is usually considered to be located in the upper or middle group of the inguinal lymph node group, and the superior medial confluence of the anterior medial and superior saphenous veins of the superficial abdominal wall. In fact, there is no specific anatomical region. How to find and locate the SLN of the penile cancer is particularly important. At present, many clinical applications such as isosulphan 
blue(IB), methylene blue(MB), ${ }^{99 m}$ Tc-colloid sulphur or ultra-small particles of iron oxide(USPIO) are used as SLN biopsy markers for penile cancer. Tang Yong et al. used carbon nanoparticle tracer technology in his first surgery in China ${ }^{7}$. Although the use of carbon nanoparticle can cause black staining of local tissue and lymph nodes at the injection site, due to the microcharacteristics of it (carbon particle diameter is only 21 $\mathrm{nm}$ ), it is phagocytized by macrophages in lymph nodes, and it does not affect HE staining of lymph nodes. It does not affect the pathological diagnosis of patients after surgery. The local injection of penile tumors prior to surgery can make first station draining node black and easies its pick up. It plays a good role in laparoscopic inguinal SLN biopsy. I

\section{Lymph node treatment principles and lymphatic leakage}

Treatment principle: For patients with palpable lymph nodes, lymph nodes groin dissection is straight forward. For patients with FNAC positive lymph nodes before surgery, the negative side is operated first. After the end of the lymph node dissection and the wound protected, partial penectomy or total penectomy was performed. Visible blood vessels and lymphatic vessels were all ligated during surgery. When using ligation clip during laparoscopic operation, selecting the absorbable clip or trumpet ligation clip is a better option to avoid postoperative subcutaneous foreign body. At the same time, the pneumo-cutaneoum pressure must not be too high. Lymphatic leakage is one of the complications of inguinal lymph node dissection. Diagnostic criteria for lymphatic leakage: Drainage fluid is more than $50 \mathrm{~mL}$ for 3 days (or more than $100 \mathrm{~mL}$ for 2 consecutive days) from the third day after inguinal lymph node dissection and is considered to be lymphatic leakage. Treatment strategies include reduced activity and wound pressure dressing (moderate pressure) to promote lymphatic closure and the formation of collateral circulation ${ }^{8}$.

\section{Surgical decision-making}

In 2006, Tobias-Machado et al. completed the first VEIL in the world, and then the surgery was carried out in many places. ${ }^{9}$ In 2010, Zhang Xiujie et al., completed the first case of VEIL in China. ${ }^{10}$ Later, there were reports of single-port laparoscopy surgery(SILS) and robotassisted laparoscopic inguinal lymph node dissection. ${ }^{11}$ According to the lymph node metastasis of the patient and the relevant technical mastery of the doctor, the best surgical method suitable for the patient can be selected. There are several surgical options to be chosen, such as partial penectomy or total penectomy, follow-up or abandonment of inguinal lymph node dissection, bilateral or unilateral inguinal lymph node dissection (staged or simultaneously), OIL or modified OIL and bilateral inguinal lymphadenectomy (staged or simultaneously), VEIL by multi-port or single-port and partial penectomy or total penectomy (simultaneously), preservation of the large saphenous vein + resection of the laparoscopic fascia laparoscopic inguinal lymph node dissection and partial penectomy or total penectomy (simultaneously) and single-port laparoscopic + robotic assisted laparoscopic inguinal lymph node dissection (simultaneously). There are other techniques such as intra-operative lymph node tracing, which also helps to further optimize surgical methods.

The NCCN Guidelines (2017 version) mention that the number and hardness of inguinal lymph nodes are helpful in predicting survival. At the same time, it was also mentioned that nearly $20 \%$ to $30 \%$ of inguinal lymph node positive patients have positive pelvic lymph nodes. The 5-year survival rate of patients with positive pelvic lymph nodes was mostly below $10 \%$. Based on this point of view, the guidelines recommend that more than two inguinal lymph nodes positive or high-grade tumor in lymph nodes guides towards pelvic lymph node dissection.

For surgeons, innovation in the concept of surgery is particularly important. Penile cancer is one of the common malignancies. Due to different national, regional, ethnic, religious and health habits, there is a clear difference in the incidence of penile cancer. However, its overall incidence is gradually decreasing. In the western countries, penile cancer is rare. In developing countries such as Asia, South America, and Africa, the incidence rate is relatively high due to poor sanitation conditions. ${ }^{12}$ The prognosis and tumor control of VEIL will be the future research direction and hot spot. ${ }^{13}$

\section{Conclusion}

Penile cancer is showing a decreasing trend in China. The laparoscopic techniques of doctors in hospitals around the world are improving rapidly. It is reasonable to apply Video Endoscopic techniques to inguinal lymph node dissection or even pelvic lymph node dissection for penile cancer. Combined with the recommendations 
of the new guideline, the doctor's world wide should change their mindset to adopt the concept of VEIL for penile cancer.

\section{References:}

1. Tian YY, Li X, Wang HZ, et al. Comparing the effect of laparoscopic and inguinal percutaneous lymph node dissection in patients with penile cancer. Journal of Sichuan University (Medical Science Edition), 2017;48(4):640-642.

2. Wang ZP, Xu YP, Li FY, et al. Clinical analysis of 23 cases of modified inguinal lymph node dissection with penile cancer under laparoscopy. Zhejiang Journal of Medicine, 2017; 39(12):1014-1016.

3. Cheng SP, Ma X, Guo G, et al. Application of laparoscopic bilateral inguinal lymph node dissection in penile cancer surgery. Chinese Journal of Clinical Oncology, 2015(2):164-167.

4. Zou L, Lai YH, Zheng X. Application analysis of alginate dressing combined with silver ion dressing in wound healing of penile cancer inguinal lymph node dissection. Journal of Nurses Training, 2015;(21):1972-1974.

5. Wu JY, Tang Y, Yi XL, et al. Systematic evaluation of the preservation of great saphenous vein during penile cancer inguinal lymph node dissection for the reduction of postoperative complications. Journal of Modern Genitourinary Oncology, 2017, 9(4): 210216.

6. Bai ZG, Wang ZH, Dai RX, et al. Preliminary exploration and practice of surgical standardization of laparoscopic inguinal lymph node dissection. Modern Journal of Urology, 2015(11):790-793.

7. Tang Y, Cheng JW, Lu HY, et al. Application of nanocarbon tracer technique in sentinel cancer sentinel lymph node biopsy (1 case report). Journal of Guangxi Medical University, 2015, 32(4): 697698.

8. Wan ZM, Dai LJ. Exploration of wound care after penile cancer inguinal lymph node dissection. Journal of Aerospace Medicine, 2017; 28(4):513-514.

9. Tobias-Machado M, Tavares A, Jr M W, et al. Video endoscopic inguinal lymphadenectomy (VEIL): initial case report and comparison with open radical procedure. Arch Esp Urol, 2006, 59(8):849-852.

10. Zhang JX, Zhang W, Song RJ, et al. Laparoscopic inguinal lymphadenectomy of the penis cancer: a case report. Journal of Nanjing Medical University (Science and Technology), 2010(9):1375-1376.

11. Josephson D Y, Jacobsohn K M, Link B A, et al. Robotic-assisted Endoscopic Inguinal Lymphadenectomy. Urology, 2009, 73(1):167-170.

12. Qiu XK, Yan DL, Mei YH, et al. Comparison of the efficacy of laparoscopic and open penile cancer inguinal lymph node dissection. Chinese Journal of Andrology, 2016;30(9):39-43.

13. Ma SJ, Yang B, Zhang LL, et al. Laparoscopic inguinal lymph node dissection for the treatment of penile carcinoma: report of 20 cases and follow-up analysis. Journal of Modern Genitourinary Oncology, 2017; 9(5): 300-301. 